\title{
Assessment of anti-SARS-CoV-2 antibodies post- Coronavac vaccination in the Amazon region of Brazil
}

Carlos David Araújo Bichara

Amaral Costa Medicina Diagnóstica

Maria Alice Freitas Queiroz

Federal University of Pará (UFPA)

Ednelza da Silva Graça Amoras

Federal University of Pará (UFPA)

Gergiane Lopes Vaz

Amaral Costa Medicina Diagnóstica

Izaura Maria Vieira Cayres Vallinoto

Federal University of Pará (UFPA)

Cléa Nazaré Carneiro Bichara

Pará State University (UEPA)

Isabella Pinheiro Costa Amaral

Amaral Costa Medicina Diagnóstica

Ricardo Ishak

Federal University of Pará (UFPA)

Antonio Carlos Rosário Vallinoto ( $\nabla$ vallinoto@ufpa.br)

Federal University of Pará (UFPA)

\section{Short Report}

Keywords: COVID-19, SARS-CoV-2, vaccine, Coronavac, antibody

Posted Date: August 27th, 2021

DOI: https://doi.org/10.21203/rs.3.rs-611323/v3

License: (c) (i) This work is licensed under a Creative Commons Attribution 4.0 International License. Read Full License 


\section{Abstract}

The race to develop a vaccine against SARS-CoV-2 (severe acute respiratory syndrome coronavirus 2 ) has raised questions regarding the safety of immunizers, efficacy regarding the production of neutralizing antibodies, and the longevity of the immune response, especially when related to variables such as sex and age. The present study evaluated the frequency of seropositivity for anti-SARS-CoV-2 (S1 and S2) total antibodies and anti-SARS-CoV-2 (receptor binding domain - RBD - S1) neutralizing antibodies in individuals vaccinated with the immunizing agent Coronavac (Sinovac). This was a cross-sectional study involving 358 individuals divided into two groups. Group 1 consisted of 205 volunteers who were tested for antiSARS-CoV-2 total antibodies (S1 and S2); group 2 consisted of 153 individuals tested for the presence of anti-SARS-CoV-2 neutralizing antibodies (RBD-S1). Seropositivity was greater than $70 \%$ in both groups, though approximately $20 \%$ of individuals showed no antibody production. The frequency of anti-SARSCoV-2 total antibodies (S1 and S2) displayed a significantly different distribution between the sexes but not according to age. The frequency of anti-SARS-CoV-2 (RBD-S1) neutralizing antibodies was $93 \%$ in the age group from 21 to 40 years, which was significantly lower with advancing age, to $76 \%$ (41 to 60 years), $72 \%$ (61 to 80 years) and 47\% (>80 years). Our results reveal a high prevalence of anti-SARS-CoV-2 total antibodies (S1 and S2) and anti-SARS-CoV-2 (RBD - S1) neutralizing antibodies in individuals who received both doses of the Coronavac vaccine, suggesting a lower efficiency of the humoral immune response among those older than 60 years of age, which might be associated with senescence of the immune system.

\section{Introduction}

The SARS-CoV-2 (severe acute respiratory syndrome coronavirus 2) pandemic that emerged in Wuhan, China, in November 2019 [1] was accompanied by a series of concerns, questions and lessons [2]. At the same time, a race never before seen in the history of worldwide science ensued with regard to the development of a vaccine capable of eliciting neutralizing antibodies against SARS-CoV-2 and reinfections by different variants of the virus [3], conferring protection against severe cases of coronavirus disease 2019 (COVID-19) [4]. New anti-SARS-CoV-2 vaccine development platforms using inactivated virus, nonreplicating viral vector, subunit, viral-like particle, DNA and mRNA were implemented $[5,6]$, generating hundreds of records of preclinical and phase II, III, and IV clinical studies [7]. Along with proposals for new technologies for the creation of anti-SARS-CoV-2 vaccines, there were also doubts and concerns [8], especially regarding the safety and efficacy of the new platforms, which, until then, were not applied to humans [9].

Parallel to advances in our understanding of the immunological mechanisms present in SARS-CoV-2 infection and in the modulation of COVID-19, phase II and III studies showed satisfactory and promising efficacy and safety results for different anti-SARS-CoV-2 vaccine platforms [5]. This resulted in 14 vaccines in use in different countries thus far. 
In Brazil, ANVISA (National Health Surveillance Agency), a government agency responsible for regulating pharmacological and immunobiological inputs, approved definitive registration of the Pfizer and AstraZeneca vaccines in January 2021. Coronavac (Sinovac) and Janssen-CILAG vaccines are approved only for emergency use [10].

The main question and reason for the different opinions and discussions is associated with the effectiveness of mass vaccination campaigns, especially with regard to the efficiency of immunization in generating protective immunity and immunological memory. This dilemma highlights the importance of evaluating variables such as sex and age, as the immune response can exhibit different dynamics based on sex [11] and physiological senescence of the immune system with age [12]. In this context, serological studies have been carried out to assess efficiency in generating postvaccination neutralizing antibodies at the population level [13].

The present study examined the frequency of anti-SARS-CoV-2 total antibodies specific for the S1 and S2 portions of the viral spike protein, as well as the presence of anti-SARS-CoV-2 (receptor binding domain RBD - S1) neutralizing antibodies, in two independent groups of individuals who sought care at the Amaral Costa Medicina Diagnóstica laboratory after receiving the second dose of the Coronavac vaccine.

\section{Materials And Methods}

\subsection{Studied samples}

This was a cross-sectional study in which blood samples were collected from March to April 2021 and included 358 individuals (Table 1) of both sexes (138 males and 220 females) aged between 21 and 96 years (average 66.6 years). The patients voluntarily sought care at the Amaral Costa Medicina Diagnóstica laboratory in the city of Belem, capital of the State of Para (Northern Brazil) at 30 days after their second dose of Coronavac (Sinovac Research and Development Co. Ltd/Butantan). Of the total number of individuals analyzed, we performed an anti-SARS-CoV-2 total antibody test (S1 and S2) for 205; 153 were tested for the presence of anti-SARS-CoV-2 neutralizing antibodies (RBD - S1).

This project was submitted to and approved by the Human Research Ethics Committee of the Institute of Health Sciences of the Federal University of Pará (CAAE: 31800720.1.0000.0018), in compliance with the guidelines and regulatory standards for research involving human beings. Individuals who agreed to participate in the study signed an informed consent form.

\subsection{Ethical aspects}

The study was approved by the Ethics and Research in Human Beings Committee of the Health Sciences Institute of the Federal University of Pará (CAAE: 31800720.1.0000.0018) in compliance with the guidelines and regulatory standards for research involving human beings, in accordance with the Declarations of Helsinki. 


\subsection{Antibody analysis}

Whole blood samples $(5 \mathrm{~mL})$ were collected in vacuum tubes without anticoagulant. Serum was separated by centrifugation. Investigation of anti-SARS-CoV-2 total antibodies (S1 and S2) was performed using a qualitative microparticle chemiluminescent immunoassay (CMIA) with the LIAISON® XL Analyzer automated platform (DiaSorin, Saluggia, VC, Italy) following the manufacturer's recommendations. The reference ranges were non-reagent $(<12 \mathrm{AU} / \mathrm{ml})$, indeterminate $\left(12.0^{3} \mathrm{x}<15.0 \mathrm{UA} / \mathrm{ml}\right)$ and reagent $(>15.0$ $\mathrm{AU} / \mathrm{ml})$.

Anti-SARS-CoV-2 (RBD-S1) neutralizing antibodies were detected using competitive enzyme immunoassay GenScript cPass ${ }^{\text {TM }}$ SARS-CoV-2 Neutralization Antibody Detection Kit (GenScript, FDA, EUA) following the manufacturer's protocol. The approach, also known as SARS-CoV-2 Surrogate Virus Neutralization Test (sVNT) Kit, is a faster, easier, more scalable and automatable alternative to traditional neutralizing antibody tests, such as the virus neutralization test (VNT), pseudovirus neutralization test (pVNT) and plaque reduction neutralization test (PRNT). The reference ranges used were nonreagent $(<20 \%)$, indeterminate $\left(20^{3} \times £ 29 \%\right)$ and reagent $(>30 \%)$.

\subsection{Statistical analysis}

Information on sex, age and antibody status was tabulated in Excel software. The calculation of antibody frequencies was performed by direct counting, and the significance of comparisons between groups was assessed by chi-square and $\mathrm{G}$ tests [14] using the Bioestat version 5.3 program. Differences were considered statistically significant when the $p$-value was $<0.05$.

\section{Results}

When evaluating anti-SARS-CoV-2 total and neutralizing antibodies, 270 samples (75.4\%) presented positive results, 70 (19.6\%) were nonreagent, and 18 (5.0\%) were indeterminate (Table 1).

Testing for anti-SARS-CoV-2 total antibodies (205 individuals) showed a positive result for 159 samples (77.6\%); 43 (20.9\%) were nonreagent, and 3 (1.5\%) were indeterminate (Table 1). Regarding anti-SARS-CoV2 neutralizing antibodies (153 individuals tested), 111 samples (72.6\%) presented a positive result, 27 (17.6\%) were nonreagent, and 15 (9.8\%) were indeterminate (Table 1).

The seropositivity profile (reagent $v s$. nonreagent) according to the results of both tests revealed a significantly higher value $(p=0.0022)$ in females $(80 \%)$ than in males $(68 \%$; Figure $1 A)$. A similar result was observed ( $87 \%$ vs. $68 \%$; $p=0.0041)$ for the 205 individuals who underwent testing for anti-SARS-CoV-2 total antibodies (Figure 1B). However, no significant differences between sexes were found for the frequency of anti-SARS-CoV-2 neutralizing antibodies (75\% vs. 69\%; Figure 1C). 
The seropositivity profile according to the results of anti-SARS-CoV-2 total antibodies plus anti-SARS-CoV-2 neutralizing antibodies (Figure $1 \mathrm{~A}$ ) indicated significant differences from the pooled analysis of age groups $(p=0.0084)$. The highest frequency occurred in the age group of $21-40$ years $(91 \%)$, gradually decreasing to $83 \%$ (41-60 years), 73\% (61-80 years) and 62\% (>80 years) as age increased. These significant differences were not observed when measuring anti-SARS-CoV-2 total antibodies (Figure 1B) but followed the same pattern when measuring only anti-SARS-CoV- 2 neutralizing antibodies $(p=0.0218$; Figure 1C); individuals aged 21 to 40 years showed $93 \%$ seropositivity, which decreased gradually with age to $76 \%$ ( 41 to 60 years), $72 \%$ (61 to 80 years) and $47 \%$ ( $>80$ years).

\section{Discussion}

The prevalence of seropositivity for anti-SARS-CoV-2 total antibodies and anti-SARS-CoV-2 neutralizing antibodies was evaluated in the present study in individuals vaccinated with two doses of Coronavac. The results were similar, regardless of the method used to assess humoral immunological response, including the frequency of those who did not produce antibodies. However, seropositivity values were lower than those reported by the manufacturer of the immunizing agent during phase I and II randomized clinical trials in adults, young people and elderly people over 60 years and higher than the value of vaccine efficacy reported by health care professionals in direct contact with COVID-19 patients [15]. A limitation of the present study is the lack of information on the occurrence of previous infection in vaccinated individuals, a variable that might interfere with the assessment of postvaccination seroconversion.

Recent studies have shown that the Coronavac vaccine is efficient in eliciting neutralizing antibodies [16, $17,18,19]$, which together with the present results, particularly those obtained for anti-SARS-CoV-2 neutralizing antibodies, are encouraging. Indeed, considering the percentage of positivity observed in the present study, the findings suggest that mass vaccination of the population with Coronavac can generate collective protection [20].

Overall, there are different opinions and discussions about the efficacy and efficiency of immunizations with anti-SARS-CoV-2 vaccines in relation to the potential for generating protective immunity and the persistence of immunological memory [9,21], especially with regard to variables such as sex and age. In general, the immune response exhibits distinct dynamics based on factors such as sex $[11,22]$ and immune system senescence [12]. In the present study, the frequency of anti-SARS-CoV-2 total antibodies was significantly higher in females, which corroborates the literature describing females as presenting increased inflammatory and humoral responses to COVID-19 [12].

Furthermore, with regard to seropositivity for anti-SARS-CoV-2 neutralizing antibodies, a high prevalence was observed among young adults; the lowest frequency was detected among elderly individuals, which suggests a lower efficiency of the vaccine to stimulate the humoral immune response in the elderly. This supports evidence for a functional and progressive decline in the immune system in the elderly patients [12]. Nevertheless, it is noteworthy that a recent phase I/II clinical trial study demonstrated immunogenicity after Coronavac vaccination in adults aged 60 years and older as well as its safety and tolerability [19]. It is important to emphasize that a lack of postvaccination humoral immune response 
detection does not indicate the absence of immunity to SARS-CoV-2, as the serological methods used do not assess the presence of cellular immunity (CD $4^{+}$and $\mathrm{CD} 8^{+} \mathrm{T}$ lymphocytes), which may be occur even in the absence of antibodies $[23,24]$.

\section{Conclusion}

The results presented herein demonstrate a similar pattern in the frequency of seropositivity for anti-SARSCoV-2 total antibodies and anti-SARS-CoV-2 neutralizing antibodies in individuals who received two doses of the Coronavac vaccine. However, a lower efficiency of the humoral immune response among the elderly was found, which may be associated with senescence of the immune system. It is important that this result is confirmed in follow-up studies because a third-dose booster might be necessary for this group, especially due to their greater vulnerability to the most severe clinical outcome of COVID-19.

Finally, considering the emergence of virus variants, the neutralizing antibody response after vaccination should be monitored. Our results support the execution of population-based serological studies aimed at better understanding the efficacy of vaccines approved for use in Brazil in terms of their ability to generate herd immunity against SARS-CoV-2.

\section{Declarations}

Author contributions: ACRV and CDAB conceived of the project. CDAB, IPCA, and GLV performed the laboratory analyses. CDAB, ESGA, and MAFQ performed the statistical analyses and wrote the draft of the article. ACRV, RI, CNCB and IMVCV reviewed the article.

Funding: This project was funded by the National Council for Scientific and Technological Development (CNPq), the Ministry of Science, Technology and Innovation (MCTI), and the Ministry of Health (MS); Process CNPQ 401235/2020-3; Amaral Costa Medicina Diagnóstica and Federal University of Pará Support Program for Qualified Production (PAPQ-2020). ACRV and RI are CNPq Grantees (\#301869/20170 and \#312979/2018-5).

Institutional Review Board Statement: The study was conducted according to the guidelines of the Declaration of Helsinki and approved by the Human Research Ethics Committee of the Institute of Health Sciences of the Federal University of Pará (CAAE: 31800720.1.0000.0018) in compliance with the guidelines and regulatory standards for research involving human beings. Individuals who agreed to participate in the study signed an informed consent form.

Data Availability Statement: Data are available upon request from the corresponding author.

Acknowledgments: We thank all individuals who agreed to participate in this study.

Conflicts of Interests: The authors declare that they have no competing interests.

\section{References}


1. World Health Organization. Coronavirus disease (COVID-2019) situation reports; 2020. https://www.who.int/emergencies/diseases/novel-coronavirus-2019/situation-reports/. (Accessed on 27/March/2020).

2. Cheng, C.; Wang, H.Y.; Chan, L. Multiple forms of mass anxiety in coronavirus disease-2019 pandemic. J Affect Disord. 2021,291:338-343. doi: 10.1016/j.jad.2021.05.034.

3. Rubin, R. COVID-19 Vaccines vs Variants-Determining How Much Immunity Is Enough. JAMA. 2021,325(13):1241-1243. doi: 10.1001/jama.2021.3370.

4. Corey, L.; Mascola, J.R.; Fauci, A.S.; Collins F.S. A strategic approach to COVID-19 vaccine R\&D. Science. 2020,368(6494):948-950. doi: 10.1126/science.abc5312.

5. Park, J.H.; Lee, H.K. Delivery Routes for COVID-19 Vaccines. Vaccines (Basel). 2021,9(5):524. doi: 10.3390/vaccines 9050524 .

6. Zhao, Q.; Gao, Y.; Xiao, M.; Huang, X.; Wu, X. Synthesis and immunological evaluation of synthetic peptide based anti-SARS-CoV-2 vaccine candidates. Chem Commun (Camb). 2021,57(12):1474-1477. doi: 10.1039/d0cc08265a.

7. WHO. World Health Organization. COVID-19 vaccine tracker and landscape. https://www.who.int/publications/m/item/draft-landscape-of-covid-19-candidatevaccines. (Accessed on 07/June/2021).

8. Fedele, F.; Aria, M.; Esposito, V.; Micillo, M.; Cecere, G.; Spano, M.; De Marco, G. COVID-19 vaccine hesitancy: a survey in a population highly compliant to common vaccinations. Hum Vaccin Immunother. 2021;7:1-7. doi: 10.1080/21645515.2021.1928460.

9. Blumental, S.; Debré, P. Challenges and Issues of Anti-SARS-CoV-2 Vaccines. Front Med (Lausanne). 2021,8:664179. doi: 10.3389/fmed.2021.664179.

10. ANVISA. Agencia Nacional de Vigilância Sanitárias. Vacinas - Covid-19 https://www.gov.br/anvisa/ptbr/assuntos/paf/coronavirus/vacinas (Accessed on 07/June/2021).

11. Ulhaq, Z.S.; Soraya, G.V.; Zambrano, L.E.A.; Garcia, CP. Sexual dimorphism in SARS-COV-2 infection. Acta Endocrinol (Buchar). 2020,16(4):522-523. doi: 10.4183/aeb.2020.522.

12. Ciarambino, T.; Para, O.; Giordano, M. Immune system and COVID-19 by sex differences and age. Womens Health (Lond). 2021,17:17455065211022262. doi: 10.1177/17455065211022262.

13. Edara, V.V.; Hudson, W.H.; Xie, X.; Ahmed, R.; Suthar, M.S. Neutralizing Antibodies Against SARS-CoV-2 Variants After Infection and Vaccination. JAMA. 2021,325(18):1896-1898. doi: 10.1001/jama.2021.4388.

14. McDonald, J.H. (2014). G-test of goodness-of-fit. Handbook of Biological Statistics (Third ed.). Baltimore, Maryland: Sparky House Publishing. pp. 53-58

15. Instituto Butantan. Vacina adsorvida covid-19 (inativada) https://vacinacovid.butantan.gov.br/bulas. (Accessed on 08/June/2020).

16. Rogliani, P.; Chetta, A.; Cazzola, M.; Calzetta, L. SARS-CoV-2 Neutralizing Antibodies: A Network MetaAnalysis across Vaccines. Vaccines (Basel). 2021,9(3):227. doi: 10.3390/vaccines9030227 
17. Tanriover, M.D.; Doğanay, H.L.; Akovam, M.; Güner, H.R.; Azap, A.; Akhan, S.; Köse, Ş.; Erdinç, F.Ş.; Akalın, E.H.; Tabak, Ö.F.; Pullukçu, H.; Batum, Ö.; Şimşek Yavuz, S.; Turhan, Ö.; Yıldırmak, M.T.; Köksal, İ.; Taşova, Y.; Korten, V.; Yılmaz, G.; Çelen, M.K.; Altın, S.; Çelik, I.; Bayındır, Y.; Karaoğlan, I.; Yılmaz, A.; Özkul, A.; Gür, H.; Unal, S.; CoronaVac Study Group. Efficacy and safety of an inactivated whole-virion SARS-CoV-2 vaccine (CoronaVac): interim results of a double-blind, randomised, placebo-controlled, phase 3 trial in Turkey. Lancet. 2021,398(10296):213-222. doi: 10.1016/S0140-6736(21)01429-X.

18. Cao, Y.; Yisimayi, A.; Bai, Y.; Huang, W.; Li, X.; Zhang, Z.; Yuan, T.; An, R.; Wang, J.; Xiao, T.; Du, S.; Ma, W.; Song, L.; Li, Y.; Li, X.; Song, W.; Wu, J.; Liu, S.; Li, X.; Zhang, Y.; Su, B.; Guo, X.; Wei, Y.; Gao, C.; Zhang, N.; Zhang, Y.; Dou, Y.; Xu, X.; Shi, R.; Lu, B.; Jin, R.; Ma, Y.; Qin, C.; Wang, Y.; Feng, Y.; Xiao, J.; Xie, X.S. Humoral immune response to circulating SARS-CoV-2 variants elicited by inactivated and RBD-subunit vaccines. Cell Res. 2021,31(7):732-741. doi: 10.1038/s41422-021-00514-9.

19. Wu, Z.; Hu, Y.; Xu, M.; Chen, Z.; Yang, W.; Jiang, Z.; Li, M.; Jin, H.; Cui, G.; Chen, P.; Wang, L.; Zhao, G.; Ding, Y.; Zhao, Y.; Yin, W. Safety, tolerability, and immunogenicity of an inactivated SARS-CoV-2 vaccine (CoronaVac) in healthy adults aged 60 years and older: a randomised, double-blind, placebocontrolled, phase 1/2 clinical trial. Lancet Infect Dis. 2021,21(6):803-812. doi: 10.1016/S14733099(20)30987-7.

20. Omer, S.B.; Yildirim, I.; Forman, H.P. Herd Immunity and Implications for SARS-CoV-2 Control. JAMA. 2020,324(20):2095-2096. doi: 10.1001/jama.2020.20892. PMID: 33074293.

21. Widge, A.T.; Rouphael, N.G.; Jackson, L.A.; Anderson, E.J.; Roberts, P.C.; Makhene, M.; Chappell, J.D.; Denison, M.R.; Stevens, L.J.; Pruijssers, A.J.; McDermott, A.B.; Flach, B.; Lin, B.C.; Doria-Rose, N.A.; O'Dell, S.; Schmidt, S.D.; Neuzil, K.M.; Bennett, H.; Leav, B.; Makowski, M.; Albert, J.; Cross, K.; Edara, V.V.; Floyd, K.; Suthar, M.S.; Buchanan, W.; Luke, C.J.; Ledgerwood, J.E.; Mascola, J.R.; Graham, B.S.; Beigel, J.H.; mRNA-1273 Study Group. Durability of Responses after SARS-CoV-2 mRNA-1273 Vaccination. N Engl J Med. 2021,384(1):80-82. doi: 10.1056/NEJMc2032195.

22. Jin, S.; An, H.; Zhou, T.; Li, T.; Xie, M.; Chen, S.; Chen, C.; Ying, B.; Xu, Z.; Li, X.; Li, M. Sex- and agespecific clinical and immunological features of coronavirus disease 2019. PLoS Pathog. 2021,17(3):e1009420. doi: 10.1371/journal.ppat.1009420.

23. Grifoni, A.; Weiskopf, D.; Ramirez, S.I.; Mateus, J.; Dan, J.M.; Moderbacher, C.R.; Rawlings, S.A.; Sutherland, A.; Premkumar, L.; Jadi, R.S.; Marrama, D.; de Silva, A.M.; Frazier, A.; Carlin, A.F.; Greenbaum, J.A.; Peters, B.; Krammer, F.; Smith, D.M.; Crotty, S.; Sette, A. Targets of T Cell Responses to SARS-CoV-2 Coronavirus in Humans with COVID-19 Disease and Unexposed Individuals. Cell. 2020,181(7):1489-1501.e15. doi: 10.1016/j.cell.2020.05.015.

24. Gallais, F.; Velay, A.; Nazon, C.; Wendling, M.J.; Partisani, M.; Sibilia, J.; Candon, S.; Fafi-Kremer, S. Intrafamilial Exposure to SARS-CoV-2 Associated with Cellular Immune Response without Seroconversion, France. Emerg Infect Dis. 2021,27(1):113-21. doi: 10.3201/eid2701.203611.

\section{Tables}

Table 1. Frequency of anti-SARS-CoV-2 total antibodies (S1 and S2) and anti-SARS-CoV-2 neutralizing antibodies (RBD - S1) after two doses of Coronavac in Belem, Para. 


\begin{tabular}{l|l|l|l|l|l|l|l|l|l|}
\hline le & Vaccine & Test & N & Male & Female & $\begin{array}{l}\text { Age } \\
(\text { mean/SD) }\end{array}$ & $\begin{array}{l}\text { Reagent } \\
(\%)\end{array}$ & $\begin{array}{l}\text { Indeterminate } \\
(\%)\end{array}$ & $\begin{array}{l}\text { Negative } \\
(\%)\end{array}$ \\
\hline p 1 & Coronavac & Total Antibodies & 205 & 77 & 128 & $65.5 / 14.8$ & $\begin{array}{l}159 \\
(77.6 \%)\end{array}$ & $03(1.5 \%)$ & $\begin{array}{l}43 \\
(20.9 \%)\end{array}$ \\
\hline p 2 & Coronavac & $\begin{array}{l}\text { Neutralizing } \\
\text { antibodies }\end{array}$ & 153 & 61 & 93 & $65.4 / 14.6$ & $\begin{array}{l}153 \\
(72.6 \%)\end{array}$ & $15(9.8 \%)$ & $\begin{array}{l}27 \\
(17.6 \%)\end{array}$ \\
\hline ps 1 & Coronavac & $\begin{array}{l}\text { Total and Neutralizing } \\
\text { antibodies }\end{array}$ & 358 & 138 & 220 & $65.4 / 14.7$ & $\begin{array}{l}270 \\
(75.4 \%)\end{array}$ & $18(5.0 \%)$ & $\begin{array}{l}70 \\
(19.6 \%)\end{array}$ \\
\hline
\end{tabular}

SD: Standard deviation

Figures 

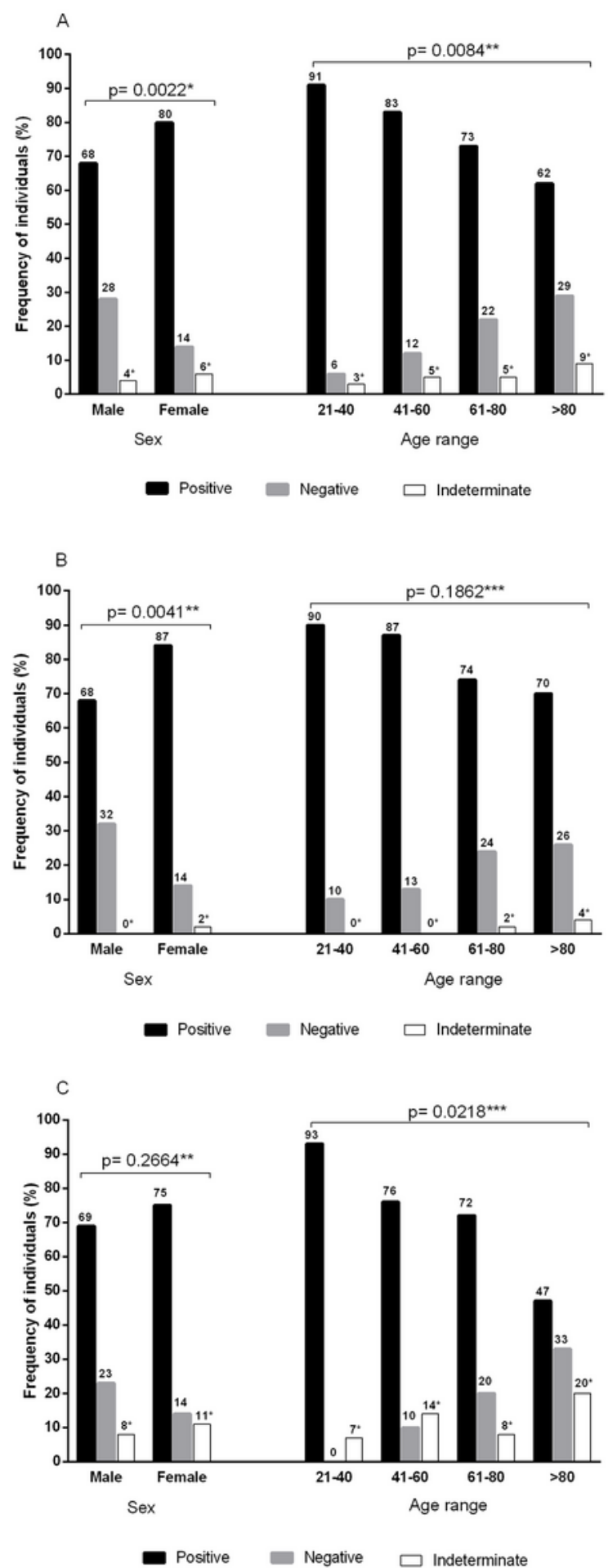

Figure 1

Frequencies of anti-SARS-CoV-2 antibodies according to sex and age group. (A) Pooled frequencies of antiSARS-CoV-2 total antibodies (S1/S2) plus anti-SARS-CoV-2 IgG neutralizing antibodies (RBD - S1). Sample size by sex: male $(n=138)$ and female $(n=220)$. Sample size by age group: $21-40(n=35), 41-60(n=60)$, 61-80 ( $n=221)$ and $>80(n=42)$. (B) Frequencies of total anti-SARS-CoV-2 antibodies (S1/S2). Sample size by sex: male $(n=77)$ and female $(n=128)$. Sample size by age group: $21-40(n=20), 41-60(n=39), 61-80$ 
$(n=119)$ and $>80(n=27)$. (C) Frequencies of neutralizing IgG anti-SARS-CoV-2 (RBD - S1) antibodies.

Sample size by sex: male $(n=61)$ and female $(n=92)$. Sample size by age group: $21-40(n=15), 41-60$ $(n=21), 61-80(n=102)$ and $>80(n=15)$. *Indeterminate results were not included in the statistical analysis;

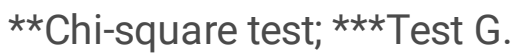

